\title{
Developing Robust Standardised Analytical Procedures for Cannabinoid Quantification: Laying the Foundations for an Emerging Cannabis-Based Pharmaceutical Industry
}

\author{
Matthew T. Welling ${ }^{a}$ Lei Liu ${ }^{a}$ Arno Hazekamp ${ }^{b}$ Ashley Dowell ${ }^{a}$ \\ Graham J. Kinga

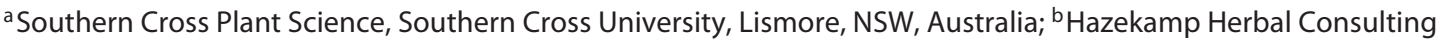 \\ BV, Leiden, The Netherlands
}

\section{Keywords \\ Cannabis sativa $\cdot$ Medicinal cannabis · Cannabidiol . \\ Tetrahydrocannabinol · Quantitative analysis · Mass spectrometry}

\begin{abstract}
The plant genus Cannabis is a prolific producer of unique pharmaceutically relevant metabolites, commonly referred to as cannabinoids. Robust and standardised methods for the quantification of cannabinoids within botanical and drug forms is a critical step forward for an emerging Cannabis-based pharmaceutical industry, which is poised for rapid expansion. Despite a growing body of analytical methods for the quantification of cannabinoids, few have been validated using internationally accredited guidelines. Moreover, standardised methods have yet to be developed for application at various stages of manufacture as well as for different levels of processing and refinement. Validation parameters for establishing robust standardised methods for cannabinoid quantification within Cannabis-based drug forms are critically discussed. Determining an appropriate level of specificity (discrimination) among heterogeneous botanical matrices as well as evaluating accuracy (recovery) and inter-labo-
\end{abstract}

ratory precision (reproducibility) within strict and volatile regulatory environments are potential obstacles to the establishment of robust analytical procedures. We argue that while some of these challenges remain unique to Cannabis, others are common to botanical-based drug development and manufacture. In order to address potential barriers to analytical method standardisation, a collaborative research initiative inclusive of academic and commercial stakeholders is proposed.

(c) 2019 The Author(s) Published by S. Karger AG, Basel

\section{Introduction}

Cannabis is a chemically complex [1], pharmaceutically relevant [2], domesticated [3], monospecific genus within the angiosperm family Cannabaceae, order Rosales [4]. Plants of this genus produce a group of isoprenylated resorcinyl polyketides, also known as cannabinoids [5]. Cannabinoids vary structurally in terms of their isoprenyl residue as well as their resorcinyl alkyl side chain and moiety (Fig. 1). Nine primary isoprenyl topological arrangements or "types" of cannabinoids occur in planta [5]. The resorcinyl alkyl residue can also vary by

\begin{tabular}{ll}
\hline KARGER & $\begin{array}{l}\text { C } 2019 \text { The Author(s) } \\
\text { Published by S. Karger AG, Basel }\end{array}$ \\
E-Mail karger@karger.com & This article is licensed under the Creative Commons Attribution- \\
www.karger.com/mca & $\begin{array}{l}\text { NonCommercial-NoDerivatives 4.0 International License (CC BY- } \\
\text { NC-ND) (http://www.karger.com/Services/OpenAccessLicense). } \\
\text { Usage and distribution for commercial purposes as well as any dis- } \\
\text { tribution of modified material requires written permission. }\end{array}$
\end{tabular}

Graham J. King

Southern Cross Plant Science

Southern Cross University, 1 Military Road

Lismore, NSW 2480 (Australia)

E-Mail graham.king@scu.edu.au 
Fig. 1. Chemical structures and topological arrangements of THC- and CBD-type isoprenylated resorcinyl polyketides (cannabinoids). Blue highlights isoprenyl topological arrangement. Orange highlights resorcinyl polyketide residue, side-chain and moiety. CBD, cannabidiol; THC, delta-9-tetrahydrocannabinol.

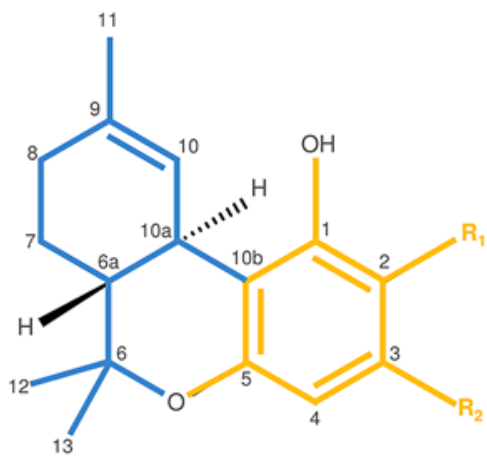

THC-type cannabinoids

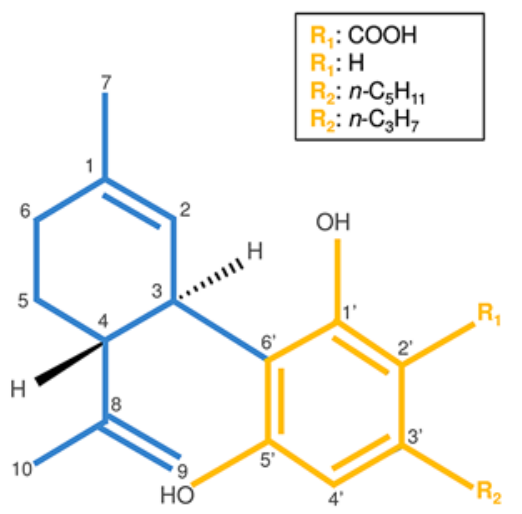

CBD-type cannabinoids carbon number [6]. Cannabinoids are synthesised with a carboxylated resorcinyl moiety that undergoes spontaneous decarboxylation via a non-enzymatic reaction, a process that can be accelerated at high temperatures [7]. More than 100 cannabinoids have been identified from Cannabis $[1,8]$. While impressive, this catalogue does not necessarily reflect the metabolic capacity of this genus but may be a consequence of oxidative instability of plantderived cannabinoids, causing a range of degradation products to occur after synthesis [5].

The current understanding of the bioactivity of cannabinoids is based on their interaction with the human endocannabinoid system, a complex network comprising of $2 \mathrm{G}$ protein-coupled receptors, a number of thermosensitive transient receptor potential cation channels, at least 2 endocannabinoids (anandamide and 2-arachidonoyl-glycerol), as well as associated biosynthetic pathways [9]. The human endocannabinoid system acts as a versatile broad-spectrum modulator of numerous biological systems and is involved in neurological and immunological pathologies $[10,11]$. Delta-9-tetrahydrocannabinol (THC) and cannabidiol (CBD), as well as their C3 alkyl cannabinoid homologues, are considered the most well studied and clinically relevant plant-derived cannabinoids (Fig. 1), with various combinatory formulations already scheduled or completed for phase 2 and 3 human clinical trials [11].

Despite the therapeutic potential of cannabinoids, the pharmaceutical application of Cannabis has been untenable for several decades. This is primarily due to a lack of high-quality clinical evidence supporting medical use [2], a situation exacerbated by legislative constraints associated with the United Nations 1961 Single Convention on Narcotic Drugs and the 1971 Convention of Psychotropic Substances, which have severely restricted research into Cannabis pharmacology and therapeutic use [3, 12]. These constraints have also inhibited clinicians from exploring therapeutic value and severely limited patient access in most legislatures, with clinical application further blocked by the removal of Cannabis from pharmacopoeias in the 1970s [13,14]. Until recently, neither of the two major central regulatory agencies which evaluate drugs, European Medicines Agency (EMA) and United States Food and Drug Administration (FDA), approved nor permitted marketing authorisations for Cannabis or Cannabis-related extracts [15]. Indeed, prior to June 2018, only two synthetic cannabinoid-based pharmaceuticals had been approved by the FDA, nabilone (Cesamet ${ }^{\circledR}$ ) [16] and dronabinol (Marinol ${ }^{\circledR}$ and Syndros $\left.{ }^{\circledR}\right)$ [17].

Changes to domestic policy relating to medicinal Cannabis have occurred rapidly in a number of countries [15]. However, there is considerable confusion with regard to the legality and accessibility of these products both within and between legislatures [15]. Marketed prescription and over-the-counter medicines approved by regulatory agencies, such as the EMA and FDA, are standardised and dosage-formulated products that have demonstrated quality, safety and efficacy for their intended use. These medicines are distinct from Cannabis-based herbal formulations, artisanal extracts and magistral preparations (pharmacist-prepared medicines) which have not been subject to quality assurances associated 
with regular drug marketing authorisation. Many of these products also require inhalational routes of administration (vaporisers/e-cigarette style inhalers) or other means of administration that are poorly characterised in relation to health outcomes [18].

A number of countries, including Israel, Canada, Germany, and the Netherlands, along with approximately half of the USA at the state level, have changed their regulations to allow patient access to medical Cannabis and related products [15]. At the decentralised national level, 23 European countries have allowed authorisation of the oromucosal spray nabiximols $\left(\right.$ Sativex ${ }^{\circledR}$ ) for multiple sclerosis-associated indications [15]. In Australia, medicinal Cannabis products were nationally rescheduled to a Schedule 8 Controlled Drug in November 2016. In order for medicinal Cannabis products to be lawfully supplied in Australia, they require entry into the Australian Register of Therapeutic Goods (ARTG), an example being nabiximols (Sativex ${ }^{\circledR}$ ) (ARTG ID: 181978). Cannabis medicinal products which lack sufficient quality assurances to appear in the ARTG can currently be obtained through special access schemes (www.tga.gov.au).

The World Health Organization (WHO)'s Expert Committee on Drug Dependence met in June 2018 for a full review on the medicinal use of Cannabis and other Cannabis-derived substances in response to a series of landmark studies which provide support for the efficacy of CBD in treatment-resistant epilepsy $(n=214)$ [19], Dravet $(n=120)$ [20] and Lennox-Gastaut syndromes $(n=171)$ [21]. This coincides with the recent approval of the CBD-based drug Epidiolex ${ }^{\circledR}$ for the treatment of seizures associated with severe forms of epilepsy (Dravet and Lennox-Gastaut syndromes) [22], with Epidiolex ${ }^{\circledR}$ being the first Cannabis-derived drug to be approved by the FDA or any central regulatory agency responsible for drug evaluation.

Drug development of Cannabis is poised for rapid expansion, as can be seen by the growing portfolio of chemical phenotypes for therapeutic end-use $[23,24]$ as well as the proliferation of patent applications relating to Cannabis-based pharmaceutical preparations, delivery technologies and medical treatments [25]. Despite the promising global outlook for Cannabis-based drug development, a critical step forward for this emerging industry is the establishment of robust standardised methods for the quantification of cannabinoids within a wide and growing range of Cannabis-based drug forms [26-29]. Refinement of these methodologies and standardisation of approach will be necessary to establish a foundation for future drug development, application and manufacture and

Robust Standardised Cannabinoid Analytical Procedures will be required to ensure the identity, efficacy, purity, quality and potency of impending pharmaceutical-grade drugs derived from Cannabis.

\section{Cannabis Drug Forms}

For a botanical form of Cannabis to be suitable for marketing as a prescription drug, it must be approved by an authoritative regulatory agency (FDA, EMA, Australian Therapeutic Goods Administration, etc.). A Cannabis drug product is a finished dosage drug in a form that consists of the major active ingredient and potentially other minor active ingredients which may have modulatory function (drug substance) together with excipients (Fig. 2). Cannabis drug products can fall into one of two definitions, depending on the level of purification of the active ingredient (Fig. 2). The official term botanical drug product was developed by the FDA to describe drug products containing and manufactured from plant materials and is similar in definition to the term herbal medicinal product used by EMA [30]. Highly purified or chemically modified botanical forms are not recognised under the definition of botanical drug and are, therefore, considered as conventional drug products (Fig. 2). Cannabisderived non-prescription over-the-counter botanical drugs can also be approved by regulatory authorities, such as the FDA, with the provision that clinical safety and efficacy has been sufficiently established.

The first stage of Cannabis-derived drug product manufacture involves the harvesting of mature female racemes (inflorescences) which are cut at the base [31] and dried in temperature- and humidity-controlled environments [32]. After removal of stems, dried folium cum flore (leaf with flower) botanical raw material can be further sorted into low- (foliar) and high- (floral) cannabinoidcontaining tissues. In some legislatures, the latter is dispensed for use as a crude herbal preparation (flos). In accordance with Good Agricultural Practices and Good Manufacturing Practice [31], the botanical raw material is required to be subject to a number of tests relating to authentication and specification, as well as microbial, heavy metal, pesticide and aflatoxin contamination $[31,32]$.

Additional analyses to consider include tests for adulterants, including tobacco and synthetic cannabinoids, as well as tests for radioactivity if the material is suspected of being cultivated in a radioactively contaminated environment [33]. Production and quality control of Cannabis-based biological medicines derived from recombinant 


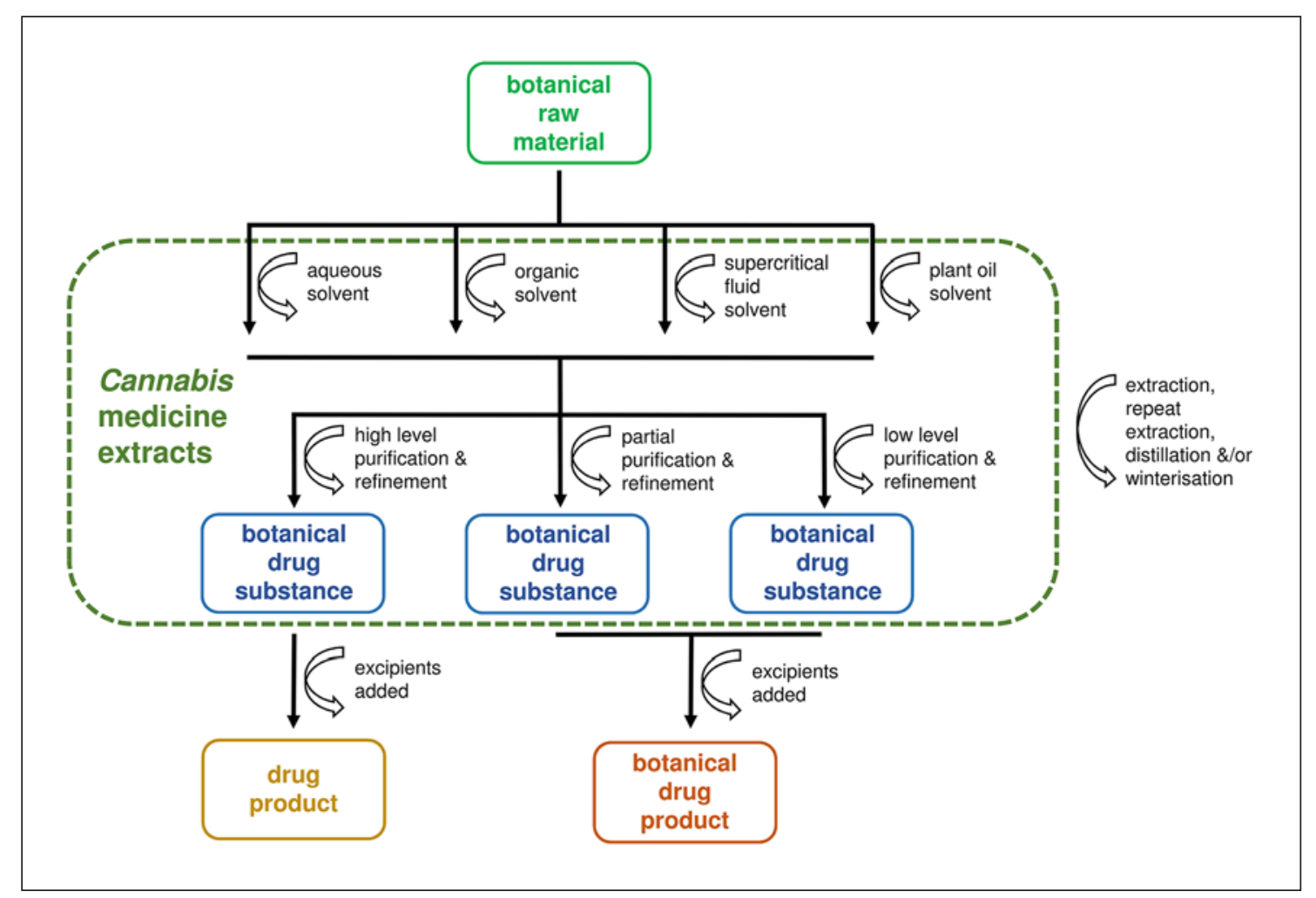

Fig. 2. Overview of Cannabis-based drug development and manufacture.

DNA technology are also subject to market authorisation guidelines (e.g., EMA European Guidelines 3AB1a), which require product purification to remove protein and nucleic acid contaminants derived from the host cell. Given the obligate outcrossing nature of Cannabis [34] as well as heterozygosity within the gene pool $[3,35]$, seedgrown Cannabis botanical raw material can be highly variable in content and composition of target and nontarget active ingredients $[31,36]$. Thus, clonal propagation using callus- [37] or nodal- (axillary buds) [38] mediated regeneration is favoured over seed propagation [22], with explants typically grown under environmentally controlled conditions in an effort to minimise genetic by environment interactions and to maximise product uniformity [31].

Cannabis medicine extracts (CME) are synonymous with botanical drug substances of Cannabis (Fig. 2). CME are derived from Cannabis botanical raw material and contain the active pharmaceutical ingredient (cannabinoids) at various levels of purification and refinement (Fig. 2). Enrichment of active ingredients for manufacture of Cannabis-based drugs can be achieved using aque- ous (decoction) [39], organic (e.g., ethanolic) [40], supercritical fluid [41, 42] and edible plant oil-based extraction [43] (Fig. 2), with aqueous being the least appropriate given the low solubility of cannabinoids in water [44, 45]. Purification of the botanical drug substance may involve repeated extraction, chromatographic separation, distillation, as well as winterisation, whereby non-target lipidsoluble materials, such as waxes, are removed by filtration at $-20^{\circ} \mathrm{C}$ [46].

The drug product Sativex ${ }^{\circledR}$ is obtained by combining partially purified THC and CBD with botanical drug substances [47]. The final botanical drug product incorporates more than 30 other cannabinoid, terpenoid, fatty acid and sterol constituents at various concentrations and is formulated with ethanol (drug vehicle), propylene glycol (co-solvent), as well as peppermint (flavour enhancer) excipients. This is delivered as a $100 \mu \mathrm{L}$ standardised dose containing $2.7 \mathrm{mg}$ THC and $2.5 \mathrm{mg}$ CBD. In contrast, the drug product Epidiolex ${ }^{\circledR}$ uses a highly purified CBD botanical drug substance and is dose formulated with sesame oil, anhydrous ethanol, sweetener and strawberry flavouring at a concentration of $100 \mathrm{mg} / \mathrm{mL}$. 
Botanical drug registration requires the same level of safety and efficacy evaluation, and similar levels of stringent quality controls, as most conventional drug products which are highly purified, synthetic or chemically modified [48]. Indeed, for botanical drugs being used in new and investigational drug applications, the Botanical Drug Development Guidance for Industry provided by the FDA recommends a declaration of the quantitative absolute dry weight description both for the active constituents as well as other chemical constituents that are known and measurable. Unlike conventional drugs, which are typically comprised of a single active ingredient, botanical drugs can contain multiple active ingredients with specific combinatory formulations being a defining characteristic [22, 31, 47]. Therefore, quantitative characterisation of cannabinoids and other active constituents present within Cannabis drug products and substances are required not only for purposes of safety and efficacy regarding human health $[31,32]$, but also for the protection of intellectual property rights. For the latter, it is important to ensure that the botanical drug is accurately described upon registration and that the exact composition is maintained during production. In the case of drug products derived from Cannabis, accurate quantification of botanical forms will be critical for quality control through the manufacturing pipeline from botanical raw material to the refinement of botanical drug substances and development of the final drug product (Fig. 2).

\section{Validation Characteristics for Analytical Procedures}

Analytical method validation or method performance qualification is a measure of how suitable and robust an assay is over the expected concentration range at which the analyte will be analysed and provides assurance that the assay is fit for purpose. Guidance on analytical method validation for pharmaceutical materials is provided from a number of sources, such as the International Conference on Harmonisation (ICH) (document Q2 [R1] [49]), domestically relevant pharmacopeia (United States/ European, etc.) and from local government agencies, such as the FDA. No one source of validation criteria is considered superior to another or applicable to every scenario, nor are these guidelines static over time. Multiple sources of validation criteria require some level of customisation [50], which will largely depend upon the intended application of the method as well as on the jurisdiction-specific requirements of a given locality.

Robust Standardised Cannabinoid Analytical Procedures
Despite the publication of more than 130 methods for the analysis of cannabinoids between 1990 and 2016 [51], few examples exist which have been validated using internationally recognised guidelines, such as those of the $\mathrm{ICH}$ [26]. Whilst a large number of analytical techniques have been employed for the quantification of Cannabis botanical raw material [28], including the monograph Cannabis Flos from the Dutch Office of Medicinal Cannabis, limited attention has been given to the quantification of cannabinoids within Cannabis botanical drug substances or CME [27-29, 52]. This is of significant concern given the effects different extraction protocols could have on sample matrices, the chemical composition of active ingredients [27], storage requirements [45, 53] and subsequent quantitative assessment of cannabinoids. The following sub-sections discuss a validation scheme compliant with the ICH Q2 (R1) guideline and highlight generalised validation parameters for the quantification of cannabinoids within CME, with emphasis on potential challenges for fulfilling these criteria. Evaluation of these criteria informs the development of all aspects of the analytical procedure from sampling, extraction and handling of the starting material through to the optimisation of parameters associated with the final chemical analysis.

\section{Specificity (Discrimination)}

Specificity refers to the capacity to evaluate an analyte within a sample matrix [49], with the sample matrix referring to all components of the botanical drug substance and/or drug product other than the target analyte. Specificity has consequences for identification and differentiation between compounds having close homology, as well as for purity testing and for quantitative determination [49]. Not all analytical procedures across the manufacturing pipeline require a high level of specificity (complete discrimination) $[32,54]$, providing they can be compensated by one or more supporting analytical procedure(s) [49]. For example, thin layer chromatography is suitable for quantitative and semi-quantitative assessment of cannabinoids within botanical raw material $[32,54]$. However, more discriminatory procedures, which include targeted analysis on a larger portfolio of cannabinoids as well as interfering materials, are typically used during purification of drug forms in the manufacturing pipeline [31] (Fig. 2).

Chromatographic methods for identification of cannabinoids within botanical forms of Cannabis have been comprehensively evaluated in the literature $[28,55]$. The two leading analytical procedures used for cannabinoid analysis, liquid chromatography (LC) and gas chroma-

Med Cannabis Cannabinoids 2019;2:1-13 
tography (GC), have a number of trade-offs with regard to specificity, sensitivity, accuracy, precision and sample throughput. The decision to select one methodology over another is typically informed by a well-defined and clearly understood objective of the procedure. For GC, liquid samples are heated at high temperatures in the injector port during vaporisation, resulting in thermal decarboxylation of the cannabinoid resorcinyl moiety [7] (Fig. 1). As a result, GC requires prior sample derivatisation, such as a silylation, to discriminate between carboxylated cannabinoid precursors and decarboxylated cannabinoids present in the sample. This may not only provide GC with a higher degree of chromatographic resolution than LC, but the derivatization process may also allow discrimination of isomeric cannabinoid species on the basis of silyl group number [56]. In contrast, LC does not require high temperatures for chromatographic separation, allowing for identification of native carboxylated cannabinoids within botanical forms $[26,57]$. LC may also be more appropriate for quantitative cannabinoid determinations as compared with GC, as the derivatisation process cannot always be guaranteed to be exhaustive $[56,58,59]$.

For identification, ICH guidelines recommend that analytical tests should have the capacity to discriminate between structurally similar compounds that are likely to be present and that the decision to test for false positives with interfering materials should be established on sound scientific judgement [49]. A major drawback of commonly used LC-diode array detector methods is that ultraviolet spectra cannot always differentiate between important cannabinoid structural variants, such as non-aromatic cyclisation of the isoprenyl residue (e.g., cannabidiolic acid [CBDA] vs. delta-9-tetrahydrocannabinolic acid [THCA]) or the length of the resorcinyl alkyl moiety (e.g., THCA vs. tetrahydrocannabivarinic acid [THCVA]) (Fig. 1). Baseline separation of cannabinoids also becomes increasingly problematic as the portfolio of target cannabinoids increases, as has frequently been reported between CBD and cannabigerol (CBG) as well as tetrahydrocannabivarin (THCV) $[26,36,57]$. This situation is likely to be exacerbated with the analysis of complex botanical drug substances, given the more extensive variation in cannabinoid concentration within CME $[28,52]$.

Discrimination between analytes can be more accurately resolved using mass spectrometry (MS) detection on the basis of mass-to-charge ratio $(\mathrm{m} / \mathrm{z})$, with baseline separation achievable using methodologies such as selected ion monitoring, which allow for simultaneous detection of cannabinoids and associated ions that coelute [36]. However, nominal mass measurements from single mass detectors have limited discriminatory functionality for isomeric compounds $[60,61]$, such as delta-8- and delta-9-THC. LC-MS/MS with triple quadrupole, quadrupole-time-of flight (Q-TOF) [27, 61, 62] and Orbitrap [63] mass analysers can provide sufficient mass resolution of both parent and fragment ions to allow for discrimination of molecules with identical nominal mass but different elemental arrangements $[60,61]$.

Recent non-targeted profiling of CME using high-resolution LC-MS/MS has revealed a complex chemical fingerprint from ethanolic and plant oil-based extracts, with chemical composition and content dependent not only on the starting material but also on the procedure used for extraction [27]. Other reports that at least five cannabinoid species exhibit a $\mathrm{m} / z$ of 315.2294 emphasise the importance of developing analytical procedures with a high level of inherent selectivity [28]. A high level of discrimination is particularly relevant for targeted medical applications which rely on structure-activity relationships of a specific cannabinoid species in order to produce the desired physiological response following pharmaceutical exposure. For example, S,S stereochemical isomers of CBD metabolites and their dimethylheptyl analogues have been demonstrated to have potent human $G$ protein-coupled cannabinoid type-1 receptor binding compared with $\mathrm{R}, \mathrm{R}$ isomers which had very weak or no binding capacity [64]. Isomeric cannabinoids may also have different stability profiles, as is the case for THCA-A compared with THCA-B. Here, the positioning of the resorcinyl $\mathrm{COOH}$ at C-4 of THCA-B affects intramolecular hydrogen bonding, which may result in improved stability and crystallisation behaviours $[65,66]$ (Fig. 1). The potential for adulteration of botanical raw material with synthetic cannabinoids [67] is also a concern where analysis relies on methods with inherent low discrimination, such as Fourier-transform infrared (FTIR) spectroscopy. Due to the relatively large molecular size of cannabinoids, key structural features that appear between 500 and 1,500 $\mathrm{cm}^{-1}$ are difficult to interpret, making the differentiation of cannabinoid species problematic from FTIR spectra alone [68].

\section{Accuracy (Recovery)}

Accuracy is the measurement of how close the experimental value is to that of the "true" or accepted reference value of an analyte within a sample matrix. This description of accuracy is also referred to as trueness. Several methods for assessing accuracy within drug substance and drug product analytical procedures are acknowledged in the ICH Q2 (R1) [49]. In the case of drug sub- 
stances, analytical procedures can be compared (1) against reference material (analytical standards), (2) other well-characterised independent procedures, and (3) inferred from the measurement of precision, linearity and specificity [49]. In addition to methodologies (2) and (3), drug product analytical procedures can be compared using synthetic matrices spiked with known quantities of the excipients or target analyte (standard addition) [49].

Historically, legislative restrictions on the possession, manufacture and supply of certified cannabinoid reference standards have contributed to limiting the comparative assessment of accuracy. They have also limited the availability of sufficient quantities for determining spike recovery. Potential workarounds which have been applied to Cannabis include spiking certified reference standards into blank solvent exhausted botanical matrices at levels below the upper range of the analytical procedure [69] or spiking certified reference standards into small quantities of blank matrices which are potentially nonrepresentative of the botanical form (i.e., $\leq 25 \mathrm{mg}$ ) [70]. A third workaround involves gravimetrically spiking inhouse pentane extracts of botanical raw material $[26,57]$. In the latter example, the "true" reference values of the spiked concentrated Cannabis extracts were assessed using an empirical procedure (standard addition), with true values being resolved when determinations of three extract masses dissolved in solution demonstrated linearity within the calibration range [26]. Another issue with the sourcing of certified reference standards for spiked recovery is the authentication of purity. This is a problem most notable with unstable carboxylated cannabinoids, such as THCA, which rarely exist without some level of contamination [65] and, moreover, readily degrade under normal handling conditions [65, 71].

A similar procedure involving spiked recovery for the evaluation of accuracy (recovery) could be applied to CME, whereby preparative high-performance LC-based fractionation of the botanical drug substance could yield blank sample matrices and purified extracts of cannabinoids for spiking botanical drug forms. However, the inhouse development of highly purified reference materials may also be constrained by the maximum quantities permitted by regulatory authorisations imposed on research and analysis laboratories. This becomes a practical limitation considering that (1) a minimum of $1 \mathrm{~g}$ blank matrix is considered representative of the botanical form and its associated putative adsorption sites and cellular interferences [26], (2) that cannabinoids could yield greater than $73 \% w / w$ of the botanical drug substance or CME [52], and (3) that the assessment of recovery should cover the range of the analytical procedure [49]. Another consideration is that during the process of cannabinoid fractionation, the CME matrix could be altered to an extent whereby it is no longer representative. In these circumstances, extracts of the related species Humulus lupulus (hops, family Cannabaceae) [56] or Urtica dioica (family Urticaceae) [57] could be used as surrogate matrices. The use of botanically related materials is likely to be a suboptimal proxy for CME matrices, although this approach may be necessary given the rarity of Cannabis germplasm devoid of cannabinoids [72].

Irrespective of physical adsorption and interference brought about by CME matrices, matrix-dependent effects on ionisation efficiency when using MS detection can significantly affect accuracy at the level of detection. The so-called matrix effect is predominantly associated with liquid phase- [73] and electrospray ionisation-based infrastructure [74] and results in a signal non-proportional to the calibrators. Indeed, both the FDA and EMA guidelines for method validation require assessment and reduction for suppression of ionisation in MS-based analytical procedures $[75,76]$. Structural analogues can be used to mitigate ionisation efficiency as well as matrixinduced ion suppression [53] or enhancement [54]. However, they may lack sufficient structural similarity to coelute and provide full compensation. This can potentially amplify ionisation efficiency-associated error in the method as the non-coeluting analogue provides another opportunity for ion suppression/enhancement to influence methodological determinations and quantitative assessment of the target analyte $[74,75]$.

Isotopically labelled (e.g., deuterated) internal standards allow for more precise measurement of ionisation efficiency and recovery of analytes $[75,76]$. However, in some studies, the more commonly available deuterated forms have been found to have altered physiochemical properties to those of the target analyte, potentially having an impact on extraction recovery [77]. In these circumstances, ${ }^{13} \mathrm{C}$ and ${ }^{18} \mathrm{O}$ isotopically labelled internal standards would be more appropriate in LC-MS-based cannabinoid quantification, although these standards have limited availability and are typically expensive [75, 76]. Combined with the number of cannabinoids which could be present within heterogeneous CME [27], this presents a challenge for laboratories to source a comprehensive set of isotopically labelled internal standards for the purposes of method validation. Universally labelling biological samples of Cannabis through cultivation under ${ }^{13} \mathrm{C}$ conditions offers one viable option to generate a sufficient quantity and metabolic range of isotopically la- 


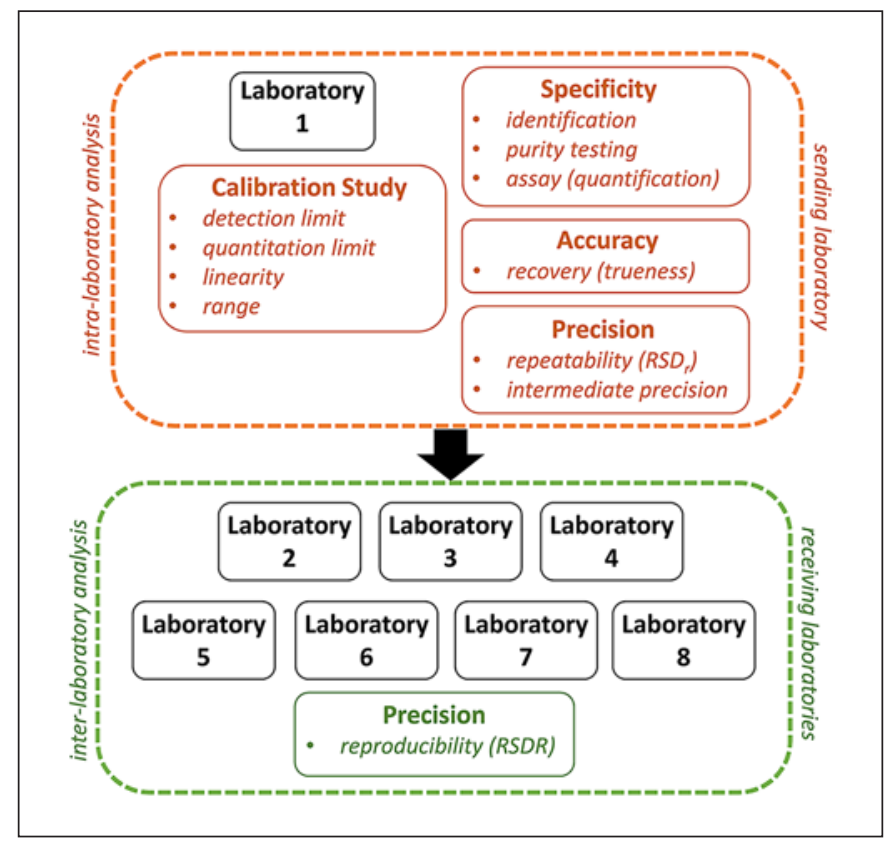

Fig. 3. Schematic diagram outlining a proposed validation scheme for intra- and inter-laboratory analytical procedures.

belled reference materials for matrix effect compensation, as well as for the measurement of accuracy and spiked recovery [78]. The generation of ${ }^{13} \mathrm{C}$ plants could be performed in-house or outsourced to companies, such as IsoLife BV (Wageningen, The Netherlands).

\section{Precision (Reproducibility)}

Precision evaluates the degree to which measurements of a homogeneous sample vary under a given condition (coefficient of variation) and is generally comprised of three levels; repeatability (relative standard deviation (RSD)r), intermediate precision and reproducibility (RSDR) [49] (Fig. 3). Repeatability and intermediate precision are intra-laboratory evaluations. Repeatability evaluates the coefficient of variation within a narrow period of time (intra-day variation), while intermediate precision assesses the coefficient of variation against random events and may include the evaluation of inter-day precision and/or the assessment of precision between analysts [49]. Acceptance criteria of $\leq 5 \%$ RSD have typically been applied to analytical procedures for quantifying cannabinoids $[26,56,57,69]$, although RSDs as high as $15 \%$ have been reported using derivatization and GC-flame ionisation detector-based analysis [59]. Having developed an analytical procedure which conforms to the acceptance criteria, the measurement of precision can be applied to Cannabis drug forms at key stages in the manufacturing pipeline in order to establish homogeneity of active constituents. Given the heterogeneous nature of botanical drugs, the FDA's Botanical Drug Development Guidance for Industry recommends that potency testing and quantification of active constituents be conducted on a batchto-batch basis.

The legitimacy of cannabinoid determinations among laboratories is a contentious subject and one which is becoming increasingly acknowledged within the literature $[29,52,79]$. The evaluation of reproducibility involves an inter-laboratory collaborative trial. This is an assessment of precision suited to analytical procedure standardisation which can contribute to the development of methodologies for inclusion in pharmacopeia $[49,80]$. In a recent large-scale comparison across six laboratories, which took into account phenotypic frequency distribution patterns of THC:CBD, the authors found significant and systemic inflation of cannabinoid quantitative determinations between laboratories after controlling for plausible confounds (i.e. producers, product types etc.) [79]. Analysis of 200 oil-based CME of Cannabis varieties Bedrocan ${ }^{\circledR}$, Bedrobinol ${ }^{\circledR}$ and Bediol ${ }^{\circledR}$ prepared at ten pharmacies in Italy showed RSD $>50 \%$ among the cannabinoids tested [29], although variation from the inter-pharmacy extraction procedures could not be disentangled from batch-to-batch variation within these registered clonallypropagated plant strains.

While such assessments may reflect inter-laboratory variation, the assessment of reproducibility requires the transfer $[81,82]$ and testing of a dedicated analytical procedure with homogeneous materials [49] as opposed to round-robin audits of non-standardised procedures among laboratories [79]. The latter provides little information on the source of variation or on the conditionspecific measurement of precision required for the evaluation of reproducibility. Reproducibility is a validation parameter treated as a random variable [83] and quasiindependent of both the analytical procedure and analyte under investigation (RSDR [\%] $=2 \mathrm{C}^{-0.1505}$, with $\mathrm{C}=$ concentration as a mass fraction) [84].

The transfer of analytical procedures is required to meet international analytical technology transfer guidelines from the International Society of Pharmaceutical Engineering and the WHO. Successful transfer of an analytical procedure is heavily dependent on equipment equivalence and harmonisation among participating laboratories [82]. As such, collaborative trials for the evaluation of reproducibility are not only subject to financial 
considerations relating to analytical infrastructure [80], but also require a high level of planning and communication among sending and receiving laboratories to ensure transfer activities are sufficient for the measurement of variability against the acceptance criteria [82] (Fig. 3). In an unlikely scenario where measurements from a number of independently developed analytical procedures are congruent with inter-laboratory precision criteria, one may conclude that a sufficient level of inter-laboratory robustness has been achieved. However, in such cases, methodological bias and error would not have been accounted for, with an associated risk that non-standardised parameters could result in out-of-specification results over the life cycle of one or more of the analytical procedures.

Evaluation of reproducibility is not a requirement for drug market authorisation [49], and despite acknowledgement for the assessment of reproducibility by government agencies, such as the FDA and pharmacopeia (United States Pharmacopeia (USP), general chapter 1224, "Transfer of Analytical Procedures" [82]), the assessment of inter-laboratory precision remains largely unregulated [82]. Although not strictly relevant for the purposes of drug authorisation, standards of AOAC INTERNATIONAL [83], as well as the International Union of Pure and Applied Chemistry (IUPAC) [85], recommend that the evaluation of reproducibility be conducted using a minimum of eight laboratories (Fig. 3). A minimum number of laboratories is also identified by the International Organization for Standardization (ISO) 5725 (1994). IUPAC will consider concessions for an absolute minimum of five laboratories in exceptional cases where the procedure involves expensive and highly specialised equipment [85]. Having established a cohort of laboratories for the measurement of reproducibility, those with out-of-specification measurements could be identified according to "analytical equivalence" which uses a predetermined range of acceptable values [50].

\section{Collaborative Research Initiative}

Prior to embarking on method performance qualification and the assessment of inter-laboratory precision, the life cycle management of the analytical procedure should be considered. This includes development of the analytical target profile which documents the requirements of the analytical procedure, an acceptable target level of uncertainty, as well as performance criteria required for validation [81]. Within this framework and as requested by

Robust Standardised Cannabinoid

Analytical Procedures
ICH Q12 guidelines [81], a multivariate analytical quality-by-design $(\mathrm{AQbD})$ approach could be implemented to inform development of robust analytical procedures and quality systems [86]. AQbD approaches can perform in silico robustness testing of critical method parameters (flow rate, column length, etc.) early in the development of an analytical procedure, and this workflow has been successfully applied to retention modelling for the separation of cannabinoids [87]. AQbD approaches establish a method operable design region or design space to mitigate out-of-specification results associated with trialand-error, one parameter at a time, optimisation-based approaches $[81,86]$. The former approach reduces the requirement for large and potentially costly retrospective changes to the analytical procedure during method performance qualification stages.

Clearly, given the complexities associated with qualification of method performance as well as the design, validation and transfer contributing to life cycle management of an analytical procedure [81], the establishment of robust and standardised procedures for cannabinoid determinations in CME requires a collaborative effort. Logistically, research organisations that may be eligible to participate within such an initiative would (1) hold relevant legal authorisations to conduct research on Cannabis, (2) have access to germplasm and representative Cannabis materials as well as analytical reference standards for the purposes of method development, and (3) be in a position to receive and supply Cannabis materials from/to participating stakeholders nationally and/or internationally.

The success of such a collaboration will be reliant on an agreed and predefined analytical target profile, independent of a specific analytical technique $[81,86,88]$. Important topics to resolve include: defining what the analytical procedure is to measure [88], for example the complement of cannabinoids to be included [89] and expected interfering materials [49], as well as how transferrable the analytical method is among heterogeneous CME matrices. The inclusion/exclusion of chemical constituents for quantitative assessment and potency testing within Cannabis drug forms will be application specific and dependent on a number of factors, including the genetic uniformity and chemotypic heterogeneity of breeding materials used in biopharmaceutical manufacture. Quantitative assessment may also need to extend to isomeric species (e.g., delta-9-trans-THC, delta-9-cis-THC and delta-8-THC) which are likely to be present as well as other chemical constituents potentially capable of modifying pharmacological activity [90]. Given that the production of CME may be proprietary and protected by in- 
tellectual property rights $[22,32,46]$, development of an analytical target profile relevant to industry will require engagement and collaborative dialogue between academic and commercial sectors.

\section{Conclusion}

A number of public health concerns arise in territories where legislators permit consumption of $\mathrm{CME}$ which have not been subject to standard quality assurance requirements that are typically associated with drug marketing authorisation $[15,91]$. Reports of inaccurate labelling and batch-to-batch variation of active ingredients within magistral CME preparations confirm fears that these products lack standardisation [29, $91,92]$. Being able to characterise accurately the complexity of botanical drug forms and to discriminate the efficacy of their constituents both singularly and in combination remains challenging [93]. This is highlighted by the fact that, so far, only two botanical drug forms have met the Botanical Guidance definition of a botanical drug product and received New Drug Application approval and prescription drug marketing authorisation by the FDA (Veregen ${ }^{\circledR}$ from Camellia sinensis as well as Fulyzaq ${ }^{\mathrm{TM}}$ [Mytesi $\left.^{\mathrm{TM}}\right]$ from Croton lechleri) [30]. Barriers to botanical drug approval have been associated not only with insufficient clinical evidence, but also inadequate experience in drug development programs by some drug sponsors [30].

Regardless of the level of refinement of the finished dosage form, the establishment of robust, standardised analytical procedures for CME is an essential step towards adoption of legitimate Cannabis-based botanical drug substances. This will not only allow for robust discrimination and quantification of active ingredients, but also inform what interfering materials are likely to be present within more purified botanical forms. It will furthermore help identify artefacts generated under different extraction and purification conditions. Understanding the complexity of CME through well-characterised and standardised analytical procedures will also facilitate the development of reliable but less discriminatory procedures more suited to routine analysis and may help pave the way forward for gold standard fit-for-purpose methodologies. This is in the individual and collective interest of all stakeholders, be they policy makers, regulators, drug manufacturers, clinicians, patients or their carers.

\section{Statement of Ethics}

The authors have no ethical conflicts to disclose.

\section{Disclosure Statement}

The authors have no conflicts of interest to declare.

\section{Funding Sources}

No funding was received for this study.

\section{Author Contributions}

M.T. Welling carried out a detailed literature survey and prepared the manuscript. L. Liu performed a detailed review and revision of the manuscript. A. Hazekamp performed a detailed review and revision of the manuscript. A. Dowell contributed to review and revision of the manuscript. G.J. King conceived the review topic and performed a detailed review and revision of the manuscript.

\section{References}

1 Elsohly MA, Slade D. Chemical constituents of marijuana: the complex mixture of natural cannabinoids. Life Sci. 2005 Dec;78(5):539-48.

2 Whiting PF, Wolff RF, Deshpande S, Di Nisio M, Duffy S, Hernandez AV, et al. Cannabinoids for medical use: a systematic review and meta-analysis. JAMA. 2015 Jun;313(24): 2456-73.

3 Welling MT, Shapter T, Rose TJ, Liu L, Stanger R, King GJ. A belated green revolution for Cannabis: virtual genetic resources to fast-track cultivar development. Front Plant Sci. 2016 Jul;7:1113.
4 Small E, Cronquist A. A practical and natural taxonomy for Cannabis. Taxon. 1976;25(4): 405-35.

5 Hanuš LO, Meyer SM, Muñoz E, TaglialatelaScafati O, Appendino G. Phytocannabinoids: a unified critical inventory. Nat Prod Rep. 2016 Nov;33(12):1357-92.

6 Shoyama Y, Hirano H, Makino H, Umekita $\mathrm{N}$, Nishioka I. Cannabis. X. The isolation and structures of four new propyl cannabinoid acids, tetrahydrocannabivarinic acid, cannabidivarinic acid, cannabichromevarinic acid and cannabigerovarinic acid, from Thai Can- nabis, 'Meao variant'. Chem Pharm Bull (Tokyo). 1977 Jan;25(9):2306-11.

7 Dussy FE, Hamberg C, Luginbühl M, Schwerzmann $\mathrm{T}$, Briellmann TA. Isolation of $\Delta 9$ THCA-A from hemp and analytical aspects concerning the determination of $\triangle 9$-THC in cannabis products. Forensic Sci Int. $2005 \mathrm{Apr}$ 149(1):3-10.

8 Radwan MM, ElSohly MA, El-Alfy AT, Ahmed SA, Slade D, Husni AS, et al. Isolation and pharmacological evaluation of minor cannabinoids from high-potency Cannabis sativa. J Nat Prod. 2015 Jun;78(6):1271-6. 
9 Di Marzo V, Piscitelli F. The endocannabinoid system and its modulation by phytocannabinoids. Neurotherapeutics. 2015 Oct; 12(4):692-8.

10 Aizpurua-Olaizola O, Elezgarai I, Rico-Barrio I, Zarandona I, Etxebarria N, Usobiaga A. Targeting the endocannabinoid system: future therapeutic strategies. Drug Discov Today. 2017 Jan;22(1):105-10.

11 Vemuri VK, Makriyannis A. Medicinal chemistry of cannabinoids. Clin Pharmacol Ther. 2015 Jun;97(6):553-8.

12 Nutt D. Illegal drugs laws: clearing a 50-yearold obstacle to research. PLoS Biol. 2015 Jan; 13(1):e1002047.

13 Bostwick JM. Blurred boundaries: the therapeutics and politics of medical marijuana. Mayo Clin Proc. 2012 Feb;87(2):172-86.

14 Nutt D. Medicinal cannabis: time for a comeback? Pharm J. 2014 Nov;293(7837):558-60.

15 Abuhasira R, Shbiro L, Landschaft Y. Medical use of cannabis and cannabinoids containing products - Regulations in Europe and North America. Eur J Intern Med. 2018 Mar;49:2-6.

16 Maida V, Ennis M, Irani S, Corbo M, Dolzhykov M. Adjunctive nabilone in cancer pain and symptom management: a prospective observational study using propensity scoring. J Support Oncol. 2008 Mar;6(3):11924.

17 Beal JE, Olson R, Laubenstein L, Morales JO, Bellman P, Yangco B, et al. Dronabinol as a treatment for anorexia associated with weight loss in patients with AIDS. J Pain Symptom Manage. 1995 Feb;10(2):89-97.

18 Russell C, Rueda S, Room R, Tyndall M, Fischer B. Routes of administration for cannabis use - basic prevalence and related health outcomes: A scoping review and synthesis. Int J Drug Policy. 2018 Feb;52:87-96.

19 Devinsky O, Marsh E, Friedman D, Thiele E, Laux L, Sullivan J, et al. Cannabidiol in patients with treatment-resistant epilepsy: an open-label interventional trial. Lancet Neurol. 2016 Mar;15(3):270-8.

20 Devinsky O, Cross JH, Laux L, Marsh E, Miller I, Nabbout R, et al.; Cannabidiol in Dravet Syndrome Study Group. Trial of cannabidiol for drug-resistant seizures in the Dravet syndrome. N Engl J Med. 2017 May;376(21): 2011-20.

21 Thiele EA, Marsh ED, French JA, Mazurkiewicz-Beldzinska M, Benbadis SR, Joshi C, et al.; GWPCARE4 Study Group. Cannabidiol in patients with seizures associated with Lennox-Gastaut syndrome (GWPCARE4): a randomised, double-blind, placebo-controlled phase 3 trial. Lancet. 2018 Mar;391(10125): 1085-96.

22 Chandra S, Lata H, ElSohly MA, Walker LA, Potter D. Cannabis cultivation: methodological issues for obtaining medical-grade product. Epilepsy Behav. 2017 May;70 Pt B:30212.
23 de Meijer EP, Hammond KM. The inheritance of chemical phenotype in Cannabis sativa L. (V): regulation of the propyl-/pentyl cannabinoid ratio, completion of a genetic model. Euphytica. 2016;210(2):291-307.

24 Welling MT, Liu L, Raymond CA, Ansari O, King GJ. Developmental Plasticity of the Major Alkyl Cannabinoid Chemotypes in a Diverse Cannabis Genetic Resource Collection. Front Plant Sci. 2018 Oct;9:1510.

25 Flores-Sanchez IJ, Ramos-Valdivia AC. A review from patents inspired by the genus Cannabis. Phytochem Rev. 2017;16(4):639-75.

26 Giese MW, Lewis MA, Giese L, Smith KM. Method for the analysis of cannabinoids and terpenes in Cannabis. J AOAC Int. 2015 NovDec;98(6):1503-22.

27 Citti C, Battisti UM, Braghiroli D, Ciccarella G, Schmid M, Vandelli MA, et al. A metabolomic approach applied to a liquid chromatography coupled to high-resolution tandem mass spectrometry method (HPLC-ESIHRMS/MS): towards the comprehensive evaluation of the chemical composition of Cannabis medicinal extracts. Phytochem Anal. 2018 Mar;29(2):144-55.

28 Citti C, Braghiroli D, Vandelli MA, Cannazza G. Pharmaceutical and biomedical analysis of cannabinoids: A critical review. J Pharm Biomed Anal. 2018 Jan;147:565-79.

29 Carcieri C, Tomasello C, Simiele M, De Nicolò A, Avataneo V, Canzoneri L, et al. Cannabinoids concentration variability in cannabis olive oil galenic preparations. J Pharm Pharmacol. 2018 Jan;70(1):143-9.

30 Ahn K. The worldwide trend of using botanical drugs and strategies for developing global drugs. BMB Rep. 2017 Mar;50(3):111-6.

31 Potter DJ. A review of the cultivation and processing of cannabis (Cannabis sativa L.) for production of prescription medicines in the UK. Drug Test Anal. 2014 Jan-Feb;6(1-2): 31-8.

32 Stott CG, Guy GW. Cannabinoids for the pharmaceutical industry. Euphytica. 2004; 140(1-2):83-93.

33 Confirming with TGO 93 (Standard for Medicinal Cannabis), Version 1.1, July 2018 [Internet]. TGA Health Safety Regulation. Australian Government, Department of Health; 2018 [cited 2019 Jan 3]. Available from: https://www.tga.gov.au/sites/default/files/ conforming-tgo-93-standard-medicinalcannabis.pdf.

34 Faeti V, Mandolino G, Ranalli P. Genetic diversity of Cannabis sativa germplasm based on RAPD markers. Plant Breed. 1996;115(5): $367-70$.

35 Soler S, Gramazio P, Figàs MR, Vilanova S, Rosa E, Llosa ER, et al. Genetic structure of Cannabis sativa var. indica cultivars based on genomic SSR (gSSR) markers: implications for breeding and germplasm management. Ind Crops Prod. 2017;104:171-8.
36 Welling M, Liu L, Shapter T, Raymond C, King G. Characterisation of cannabinoid composition in a diverse Cannabis sativa $\mathrm{L}$. germplasm collection. Euphytica. 2016; 208(3):463-75.

37 Slusarkiewicz-Jarzina A, Ponitka A, Kaczmarek Z. Influence of cultivar, explant source and plant growth regulator on callus induction and plant regeneration of Cannabis sativa L. Acta Biol Cracov Ser Bot. 2005;47(2): 145-51.

38 Lata $\mathrm{H}$, Chandra S, Techen N, Khan IA, ElSohly MA. In vitro mass propagation of Cannabis sativa L.: a protocol refinement using novel aromatic cytokinin meta-topolin and the assessment of eco-physiological, biochemical and genetic fidelity of micropropagated plants. J Appl Res Med Aromat Plants. 2016;3(1):18-26.

39 Hazekamp A, Bastola K, Rashidi H, Bender J, Verpoorte R. Cannabis tea revisited: a systematic evaluation of the cannabinoid composition of cannabis tea. J Ethnopharmacol. 2007 Aug;113(1):85-90.

40 Hazekamp A, Simons R, Peltenburg-Looman A, Sengers M, van Zweden R, Verpoorte R. Preparative isolation of cannabinoids from Cannabis sativa by centrifugal partition chromatography. J Liq Chromatogr Relat Technol. 2004;27(15):2421-39.

41 Perrotin-Brunel H, Kroon MC, van Roosmalen MJ, van Spronsen J, Peters CJ, Witkamp GJ. Solubility of non-psychoactive cannabinoids in supercritical carbon dioxide and comparison with psychoactive cannabinoids. J Supercrit Fluids. 2010;55(2):603-8.

42 Omar J, Olivares M, Alzaga M, Etxebarria N. Optimisation and characterisation of marihuana extracts obtained by supercritical fluid extraction and focused ultrasound extraction and retention time locking GC-MS. J Sep Sci. 2013 Apr;36(8):1397-404.

43 Casiraghi A, Roda G, Casagni E, Cristina C, Musazzi UM, Franzè S, et al. Extraction method and analysis of cannabinoids in cannabis olive oil preparations. Planta Med. 2018 Mar; 84(4):242-9.

44 Politi M, Peschel W, Wilson N, Zloh M, Prieto JM, Heinrich M. Direct NMR analysis of cannabis water extracts and tinctures and semi-quantitative data on delta9-THC and delta9-THC-acid. Phytochemistry. 2008 Jan; 69(2):562-70.

45 Pacifici R, Marchei E, Salvatore F, Guandalini L, Busardò FP, Pichini S. Evaluation of cannabinoids concentration and stability in standardized preparations of cannabis tea and cannabis oil by ultra-high performance liquid chromatography tandem mass spectrometry. Clin Chem Lab Med. 2017 Aug;55(10):155563.

46 Whittle BA, Guy G, inventors. GW Pharmaceuticals PLC, assignee. Pharmaceutical formulations. United States patent US20180042842A1. 2018 Feb 15.
Robust Standardised Cannabinoid Analytical Procedures
Med Cannabis Cannabinoids 2019;2:1-13 DOI: $10.1159 / 000496868$ 
47 Guy GW, Stott CG. The development of Sativex ${ }^{\circledR}$ - a natural cannabis-based medicine. In: Mechoulam R, editor. Cannabinoids as therapeutics. Basel: Birkhäuser; 2005. pp. 231-263.

48 Miroddi M, Mannucci C, Mancari F, Navarra M, Calapai G. Research and development for botanical products in medicinals and food supplements market. Evid Based Complement Alternat Med. 2013;2013:649720.

$49 \mathrm{ICH}$ [Internet]. Validation of analytical procedures: text and methodology Q2 (R1). Geneva, Switzerland: ICH; 2005 [cited 2018 Nov 1]. Available from: https://www.ich.org/fileadmin/Public_Web_Site/ICH_Products/ Guidelines/Quality/Q2_R1/Step4/Q2_R1__ Guideline.pdf.

50 Shabir GA. Validation of high-performance liquid chromatography methods for pharmaceutical analysis. Understanding the differences and similarities between validation requirements of the US Food and Drug Administration, the US Pharmacopeia and the International Conference on Harmonization. J Chromatogr A. 2003 Feb;987(1-2):57-66.

51 Klein RF. Analysis of marijuana by liquid chromatographic techniques - a literature survey, 1990-2015. Microgram J. 2015 Jan; 12(1-4):1-17.

52 Sexton M, Shelton K, Haley P, West M. Evaluation of cannabinoid and terpenoid content: cannabis flower compared to supercritical CO2 concentrate. Planta Med. 2018 Mar; 84(4):234-41.

53 Calvi L, Pentimalli D, Panseri S, Giupponi L, Gelmini F, Beretta G, et al. Comprehensive quality evaluation of medical Cannabis sativa L. inflorescence and macerated oils based on HS-SPME coupled to GC-MS and LC-HRMS (q-exactive orbitrap ${ }^{\circledR}$ ) approach. J Pharm Biomed Anal. 2018 Feb;150:208-19.

54 Fischedick JT, Glas R, Hazekamp A, Verpoorte R. A qualitative and quantitative HPTLC densitometry method for the analysis of cannabinoids in Cannabis sativa L. Phytochem Anal. 2009 Sep-Oct;20(5):421-6.

55 Leghissa A, Hildenbrand ZL, Schug KA. A review of methods for the chemical characterization of cannabis natural products. J Sep Sci. 2018 Jan;41(1):398-415.

56 Leghissa A, Hildenbrand ZL, Foss FW, Schug KA. Determination of cannabinoids from a surrogate hops matrix using multiple reaction monitoring gas chromatography with triple quadrupole mass spectrometry. J Sep Sci. 2018 Jan;41(2):459-68.

57 De Backer B, Debrus B, Lebrun P, Theunis L, Dubois N, Decock L, et al. Innovative development and validation of an HPLC/DAD method for the qualitative and quantitative determination of major cannabinoids in cannabis plant material. J Chromatogr B Analyt Technol Biomed Life Sci. 2009 Dec;877(32): 4115-24.
58 Hazekamp A. Cannabis: extracting the medicine. Thesis, Leiden University. 2007. Available from: https://openaccess.leidenuniv.nl/ handle/1887/12297.

59 Ibrahim EA, Gul W, Gul SW, Stamper BJ, Hadad GM, Abdel Salam RA, et al. Determination of acid and neutral cannabinoids in extracts of different strains of Cannabis sativa using GC-FID. Planta Med. 2018 Mar;84(4): 250-9.

60 Lacorte S, Fernandez-Alba AR. Time of flight mass spectrometry applied to the liquid chromatographic analysis of pesticides in water and food. Mass Spectrom Rev. 2006 Nov-Dec; 25(6):866-80.

61 Aizpurua-Olaizola O, Omar J, Navarro P, Olivares M, Etxebarria N, Usobiaga A. Identification and quantification of cannabinoids in Cannabis sativa L. plants by high performance liquid chromatography-mass spectrometry. Anal Bioanal Chem. 2014 Nov; 406(29):7549-60.

62 Marti G, Schnee S, Andrey Y, Simoes-Pires C Carrupt PA, Wolfender JL, et al. Study of leaf metabolome modifications induced by UV-C radiations in representative Vitis, Cissus and Cannabis species by LC-MS based metabolomics and antioxidant assays. Molecules. 2014 Sep;19(9):14004-21.

63 Concheiro M, Lee D, Lendoiro E, Huestis MA. Simultaneous quantification of $\Delta(9)$ tetrahydrocannabinol, 11-nor-9-carboxy-tetrahydrocannabinol, cannabidiol and cannabinol in oral fluid by microflow-liquid chromatography-high resolution mass spectrometry. J Chromatogr A. 2013 Jul;1297: 123-30.

64 Hanuš LO, Tchilibon S, Ponde DE, Breuer A, Fride E, Mechoulam R. Enantiomeric cannabidiol derivatives: synthesis and binding to cannabinoid receptors. Org Biomol Chem. 2005 Mar;3(6):1116-23.

65 McPartland JM, MacDonald C, Young M, Grant PS, Furkert DP, Glass M. Affinity and efficacy studies of tetrahydrocannabinolic acid $\mathrm{A}$ at cannabinoid receptor types one and two. Cannabis Cannabinoid Res. 2017 May; 2(1):87-95

66 Rosenqvist E, Ottersen T. The crystal and molecular structure of delta-9-tetrahydrocannabinolic acid b. Acta Chem Scand B. 1975; 29(3):379-84

67 Adams AJ, Banister SD, Irizarry L, Trecki J, Schwartz M, Gerona R. "Zombie" outbreak caused by the synthetic cannabinoid AMBFUBINACA in New York. N Engl J Med. 2017 Jan;376(3):235-42.

68 Zuba D, Geppert B, Sekuła K, Żaba C. [1-(Tetrahydropyran-4-ylmethyl)-1H-indol-3-yl](2,2,3,3-tetramethylcyclopropyl) methanone: a new synthetic cannabinoid identified on the drug market. Forensic Toxicol. 2013;31(2): 281-91.
69 Wang YH, Avula B, ElSohly MA, Radwan MM, Wang M, Wanas AS, et al. Quantitative determination of $\triangle 9$-THC, CBG, CBD, their acid precursors and five other neutral cannabinoids by UHPLC-UV-MS. Planta Med. 2018 Mar;84(4):260-6.

70 Brighenti V, Pellati F, Steinbach M, Maran D, Benvenuti S. Development of a new extraction technique and HPLC method for the analysis of non-psychoactive cannabinoids in fibre-type Cannabis sativa L. (hemp). J Pharm Biomed Anal. 2017 Sep;143:228-36.

71 Citti C, Ciccarella G, Braghiroli D, Parenti C, Vandelli MA, Cannazza G. Medicinal cannabis: principal cannabinoids concentration and their stability evaluated by a high performance liquid chromatography coupled to diode array and quadrupole time of flight mass spectrometry method. J Pharm Biomed Anal. 2016 Sep;128:201-9.

72 de Meijer EP, Hammond KM, Sutton A. The inheritance of chemical phenotype in Cannabis sativa L. (IV): cannabinoid-free plants. Euphytica. 2009;168(1):95-112.

73 King R, Bonfiglio R, Fernandez-Metzler C Miller-Stein C, Olah T. Mechanistic investigation of ionization suppression in electrospray ionization. J Am Soc Mass Spectrom. 2000 Nov;11(11):942-50.

74 Stokvis E, Rosing H, Beijnen JH. Stable isotopically labeled internal standards in quantitative bioanalysis using liquid chromatography/mass spectrometry: necessity or not? Rapid Commun Mass Spectrom. 2005;19(3): 401-7.

75 George R, Haywood A, Khan S, Radovanovic M, Simmonds J, Norris R. Enhancement and suppression of ionization in drug analysis using HPLC-MS/MS in support of therapeutic drug monitoring: a review of current knowledge of its minimization and assessment Ther Drug Monit. 2018 Feb;40(1):1-8.

76 Schuhmacher J, Zimmer D, Tesche F, Pickard V. Matrix effects during analysis of plasma samples by electrospray and atmospheric pressure chemical ionization mass spectrometry: practical approaches to their elimination. Rapid Commun Mass Spectrom. 2003; 17(17):1950-7.

77 Wieling J. LC-MS-MS experiences with internal standards. Chromatographia. 2002;55 S1:S107-13.

78 Bueschl C, Kluger B, Lemmens M, Adam G, Wiesenberger G, Maschietto V, et al. A novel stable isotope labelling assisted workflow for improved untargeted LC-HRMS based metabolomics research. Metabolomics. 2014; 10(4):754-69.

79 Jikomes N, Zoorob M. The cannabinoid content of legal Cannabis in Washington State varies systematically across testing facilities and popular consumer products. Sci Rep. 2018 Mar;8(1):4519.

80 Thompson M, Ellison SL, Wood R. Harmonized guidelines for single-laboratory validation of methods of analysis (IUPAC Technical Report). Pure Appl Chem. 2002;74(5):835-55. 
81 Parr MK, Schmidt AH. Life cycle management of analytical methods. J Pharm Biomed Anal. 2018 Jan;147:506-17.

82 Ermer J, Limberger M, Lis K, Wätzig $H$. The transfer of analytical procedures. J Pharm Biomed Anal. 2013 Nov;85:262-76.

83 AOAC International [Internet]. AOAC Guidelines for Single Laboratory Validation of Chemical Methods for Dietary Supplements and Botanicals. Gaithersburg, USA; 2002 [cited 2018 Nov 1]. Available from: https://www.aoac.org/aoac_prod_imis/ AOAC_Docs/StandardsDevelopment/SLV_ Guidelines_Dietary_Supplements.pdf.

84 Horwitz $\bar{W}$, Albert R. The Horwitz ratio (HorRat): A useful index of method performance with respect to precision. J AOAC Int. 2006 Jul-Aug;89(4):1095-109.

85 Horwitz W. Protocol for the design, conduct and interpretation of method-performance studies: revised 1994 (Technical Report). Pure Appl Chem. 1995;67(2):331-43.
86 Peraman R, Bhadraya K, Padmanabha Reddy Y. Analytical quality by design: a tool for regulatory flexibility and robust analytics. Int J Anal Chem. 2015;2015:868727.

87 Fekete S, Sadat-Noorbakhsh V, Schelling C, Molnár I, Guillarme D, Rudaz S, et al. Implementation of a generic liquid chromatographic method development workflow: application to the analysis of phytocannabinoids and Cannabis sativa extracts. J Pharm Biomed Anal. 2018 Jun;155:116-24.

88 Schweitzer M, Pohl M, Hanna-Brown M, Nethercote P, Borman P, Hansen G, et al. Implications and opportunities of applying $\mathrm{QbD}$ principles to analytical measurements. Pharm Technol. 2010 Feb;34(2):52-9.

89 Noestheden M, Friedlander G, Anspach J, Krepich S, Hyland KC, Zandberg WF. Chromatographic characterisation of 11 phytocannabinoids: quantitative and fit-to-purpose performance as a function of extra-column variance. Phytochem Anal. 2018 Sep;29(5): 507-15.
90 Tose LV, Santos NA, Rodrigues RR, Murgu $\mathrm{M}$, Gomes AF, Vasconcelos GA, et al. Isomeric separation of cannabinoids by UPLC combined with ionic mobility mass spectrometry (TWIM-MS) - Part I. Int J Mass Spectrom. 2017 July;418:112-21.

91 Hazekamp A. The trouble with CBD oil. Med Cannabis Cannabinoids. 2018;1(1):65-72.

92 Pavlovic R, Nenna G, Calvi L, Panseri S, Borgonovo G, Giupponi L, et al. Quality traits of "cannabidiol oils": cannabinoids content, terpene fingerprint and oxidation stability of European commercially available preparations. Molecules. 2018 May;23(5):1230.

93 Yang Z, Shao Q, Ge Z, Ai N, Zhao X, Fan X. A bioactive chemical markers based strategy for quality assessment of botanical drugs: xuesaitong injection as a case study. Sci Rep. 2017 May;7(1):2410 